\title{
Burst Synchronization of Slotted Random Access With Preamble Power Ramping in the Reverse Link of CDMA Systems
}

\author{
Wern-Ho Sheen, Member, IEEE, Chun-Chieh Tseng, and Jan-Shin Ho, Student Member, IEEE
}

\begin{abstract}
Efficient random access is one of the key designs in code-division multiple-access (CDMA) cellular systems. Random access is used by user equipment for initial access, requesting dedicated channels and transmitting short packet data to a base station. In random access, a preamble part is usually devised for fast and reliable burst synchronization, which is essential in order to avoid excessive access delay and/or repeated transmissions that may reduce the overall system capacity. This paper investigates the issue of burst synchronization for the slotted random access with preamble power ramping in the reverse link of CDMA systems. A flexible burst synchronizer based on a parallel-serial code-phase detector is proposed, which can be easily configured to achieve different complexity/performance tradeoffs. A general analysis is also presented with important design parameters being taken into account, including the number of correlators, power control error, power ramping step, diversity order, frequency offset, multipath combining, and others. The analysis is verified by computer simulations.
\end{abstract}

Index Terms-Burst synchronization, power ramping, random access, wideband code-division multiple-access (WCDMA).

\section{INTRODUCTION}

$\mathbf{E}$ FFICIENT UPLINK transmission on random access channel (RACH) is one of the key designs in code-division multiple-access (CDMA) cellular systems. RACH is used by user equipments (UE) for initial access, requesting dedicated channels, and transmitting short packet data to a base station. RACH often consists of a preamble part followed by a message part. Messages can be detected correctly only after burst synchronization is achieved with the preamble part [1]-[10].

During random access, closed loop power control is not practical. Instead, open loop power control is employed to set up the transmit power, where the combined path and shadowing loss of the uplink is estimated from that of the downlink. Unfortunately, the estimation is far from accurate because of measurement errors and the effect of multipath fading, and hence, a large power

Manuscript received October 2, 2001; revised April 10, 2002; accepted April 23,2002 . The editor coordinating the review of this paper and approving it for publication is R. Murch.

W.-H. Sheen is with the Department of Communication Engineering, National Chiao Tung University, Hsinchu 300, Taiwan, R.O.C. (e-mail: whsheen@cm.nctu.edu.tw).

C.-C. Tseng is with the Syncomm Technology Corps. Chung-Li, Taoyuan 320, Taiwan, R.O.C. (e-mail: rogertcc@syncomm.com.tw).

J.-S. Ho is with the Department of Electrical Engineering, National Chung Cheng University, Chia Yi 612, Taiwan, R.O.C. (e-mail: jesseho@bras.cm. nctu.edu.tw).

Digital Object Identifier 10.1109/TWC.2003.817451 margin may be required to ensure a successful random access. A large power margin, on the other hand, results in undesirable excessive multiple access interference that reduces the overall system capacity.

One popular remedy to this drawback is to use a power ramping scheme along with open loop power control [1]-[10]. The basic idea is that the first access is transmitted at a power level well below that suggested by the open loop power control, and each additional access is then transmitted at an increased power in case the previous one is not successful. Two different power ramping methods have been employed in today's CDMA systems [1], [2], [8]-[10]. One is message power ramping in which increasing power is applied to both the preamble and message parts for each new access attempt. The other is preamble power ramping, where increasing power is applied only to the preamble part, and the message part is not transmitted until the burst (preamble) is synchronized. The selection of one scheme over the other depends on the system parameters such as open loop power control error, UE mobility, traffic load, and some others [8]-[10]. In general, the preamble ramping has less interference and lower access delay, as compared to the message ramping counterpart [8]. The message and preamble power ramping have been employed in the IS-95/CDMA-2000 and 3GPP wideband CDMA (WCDMA)/frequency-division duplexing (FDD) systems, respectively.

Fast burst (preamble) synchronization is essential in random access in order to avoid excessive access delay and/or frequent retransmissions that may reduce the overall system capacity due to multiple access interference. In [8]-[14] and [15]-[17], burst synchronization was investigated for random accesses with preamble and message power ramping, respectively. In particular, for the 3GPP WCDMA/FDD system (preamble power ramping), burst synchronizers based on a matched filter were simulated in [8], [9], [11], [12], and [14], a synchronizer using fast Fourier transform algorithm was proposed in [13], and a synchronizer with surface acoustic wave matched filter was proposed and simulated in [14]. On the other hand, burst synchronizers based on parallel-serial active correlation and parallel matched filters were investigated in [15], [17], and [16], respectively, for the IS-95/CDMA-2000 system. (In [15] and [17], only the case with the same power for each packet is considered.)

In this paper, a burst synchronizer based on a flexible parallel-serial code-phase detector is proposed for random access with preamble power ramping. Numerical results show that the proposed synchronizer can be easily configured to 


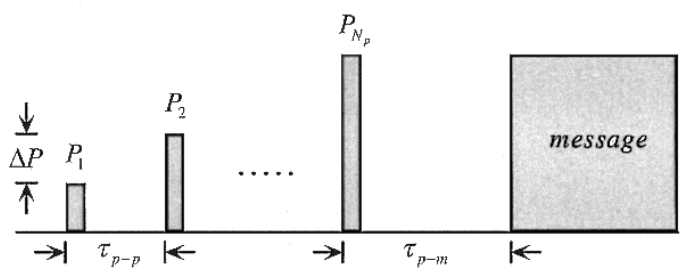

Fig. 1. Basic principle of the slotted random access with preamble power ramping.

achieve different complexity/performance tradeoffs. In addition, instead of using simulations, performance evaluation is conducted analytically, which is very general and complete to include the effects of the important parameters such as multipath combining, power control error, the maximum number of preambles, power ramping step, diversity order, frequency offset, multipath fading, and others.

This paper is organized as follows. Following this introduction, Section II discusses the system and channel models. Section III describes the proposed burst synchronizer. The performance analysis is presented in Section IV. Section V gives numerical results. Finally, Section VI summarizes this paper.

\section{SySTEM AND CHANNEL MODELS}

\section{A. System Model}

Fig. 1 is a simplified diagram illustrating the basic principle of the slotted random access with preamble power ramping. Slotted random access implies that a UE can only initiate an access at a slot boundary which is made known to the UE through synchronization to the downlink of the base station. Preambles with increased power (ramping) are transmitted successively until burst synchronization is accomplished or the maximum number of allowable preambles is reached. The power ramping step and the maximum number of preambles are denoted as $\Delta P$ (dB) and $N_{P}$, respectively. Upon burst synchronized at the base station, an acknowledgment (ACK) is sent back to notify the UE that it is ready for message reception and the message transmission can commence.

In practical systems, the time between preambles $\tau_{p-p}$ and the time between preamble and message $\tau_{p-m}$ should be long enough to allow ACK reception at the UE and to prepare the base station for the subsequent message detection, respectively [1]. In addition, different spreading codes (signatures) and/or different access slots may be used by different users in order to reduce the chance of collisions between different users [1], [2]. In this study, no error will be assumed for ACK reception, and since our main concern is on the burst synchronization by using preamble power ramping, the effect of collisions and capturing after a collision will not be considered, although it should be included in the evaluation of the throughput and delay of random access [8].

In the slotted random access, although the slot boundaries are known to a UE, there still exists a time uncertainty between the $\mathrm{UE}$ and base station due to the maximum round trip delay, which is equal to $2 d_{b-m} / c$, where $d_{b-m}$ and $c$ are the maximum distance between the base station and UE and the speed of light, respectively. In fact, determining the exact time difference between a UE and a base station is what the burst synchronization is all about. Burst synchronization is usually achieved by synchronizing the pseudonoise codes used to spread the preambles in CDMA systems.

For easy implementation, the time uncertainty is usually quantized into a number of intervals, called cells (code phases), with size equal to one chip duration. ${ }^{1}$ As a consequence, the issue of burst synchronization becomes to determine in which cell a preamble has arrived. In the following, the cell $\mathcal{C}_{i}$ is to denote the $i$ th interval, from the beginning of the time uncertainty, that the preamble can arrive, and the total number of cells is equal to $N_{u}$. In addition, the true cells in which the preamble has arrived will be denoted as the $H_{1}$ cells and all others are the $H_{0}$ cells. More than one $H_{1}$ cell is possible for multipath channels.

\section{B. Signal and Channel Model}

The signal format of the $p$ th transmitted preamble is given as follows:

$$
\begin{array}{r}
u(t)=\sqrt{2 E_{c}^{(p)}} \sum_{k=0}^{K-1} h\left(t-k T_{c}\right) \operatorname{Re}\left[c_{k} \exp \left(\boldsymbol{j} 2 \pi f_{c} t\right)\right] \\
p=1 \ldots N_{P}
\end{array}
$$

where $\boldsymbol{j} \doteq \sqrt{-1}, K$ is the total number of chips in a preamble, $h(t)$ is the chip shaping function with unit energy, $T_{c}$ is the chip period, $f_{c}$ is the carrier frequency, $c_{k}$ is the complex preamble code with $\left|c_{k}\right|=1, \operatorname{Re}[\cdot]$ is the real part of $\cdot$, and $E_{c}^{(p)}$ is the chip energy. Note that $E_{c}^{(p)}=E_{c}^{(p-1)}+\Delta P(\mathrm{~dB}), 1<p \leq N_{P}$, and $E_{c}^{(1)}$ is modeled as a truncated $\log$ normally distributed variable with standard deviation $\sigma_{\Psi}$ decibels, due to the open-loop power control error.

A wide-sense stationary uncorrelated scattering $L$-ray channel with $D$ diversity branches is used to model the frequency-selective fading. With this model, the received signal at the front end of the $d$ th antenna is given by

$$
\begin{aligned}
r^{(d)}(t)= & \sqrt{2 E_{c}^{(p)}} \sum_{l=1}^{L} \sum_{k=0}^{K-1} h\left(t-k T_{c}-\tau_{l}^{(d)}\right) \\
& \cdot \operatorname{Re}\left[c_{k} g_{l}^{(d)}(t) \exp j 2 \pi\left(f_{c}+\Delta f\right) t\right] \\
& +I^{(d)}(t), \quad d=1, \ldots, D
\end{aligned}
$$

where $L$ is the number of the rays, $\Delta f$ is the frequency offset, and $\tau_{l}^{(d)}$ is the time delay, which is assumed to be constant over the time for synchronization. $g_{l}^{(d)}(t)$ are the Rayleigh fading and independent of each other with independent identically distributed (i.i.d.) statistics. Rays of different delay may have different variances, though.

For later use, we define ${ }^{2}$

$$
g_{l}(t) \doteq g_{c, l}(t)+j g_{s, l}(t)
$$

\footnotetext{
${ }^{1}$ In practice, the cell size might be less than one chip. In this case, the analysis is only a little more complicated than the one here, as discussed in [15] and [18].

${ }^{2}$ For simplicity of notation, the diversity branch index $d$ will be omitted whenever possible.
} 


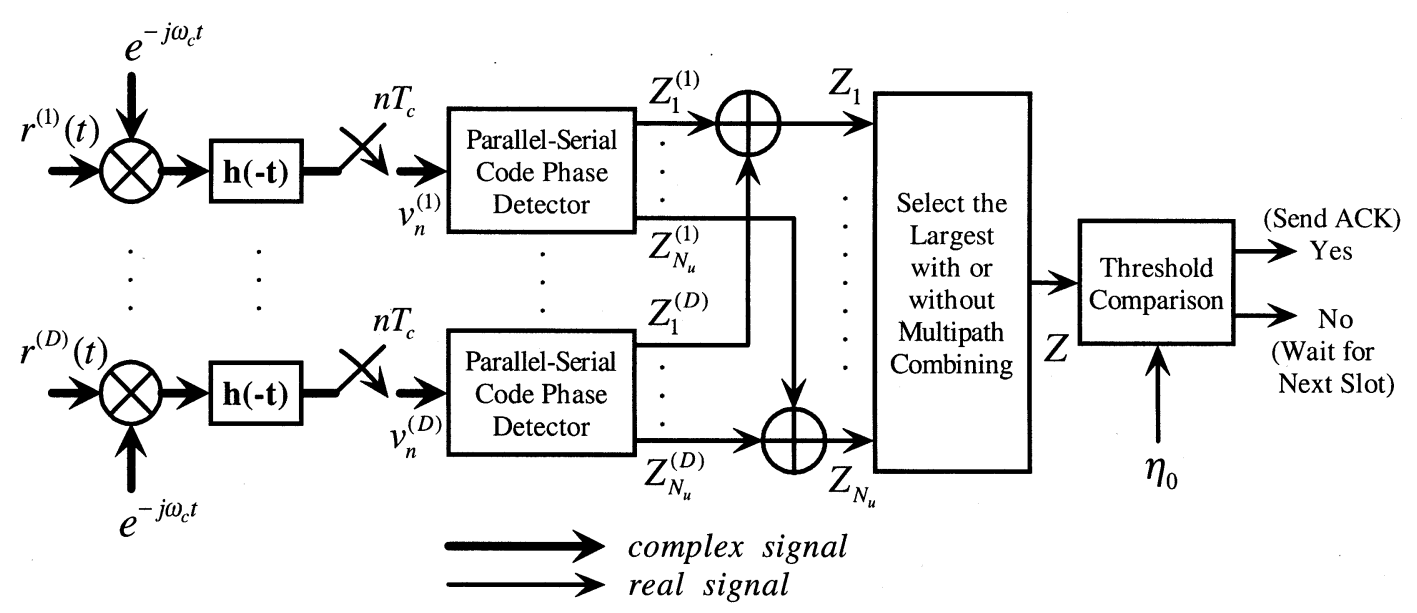

Fig. 2. Block diagram of the proposed burst synchronizers.

where $g_{c, l}(t)$ and $g_{s, l}(t)$ are i.i.d. zero mean Gaussian processes. Using the two-dimensional isotropic scattering model [19], the autocorrelation function of $g_{c, l}(t)$ and $g_{s, l}(t)$ is given by

$$
\begin{aligned}
R_{g_{l}}(\tau) & \doteq E\left[g_{c, l}(t) g_{c, l}(t+\tau)\right]=E\left[g_{s, l}(t) g_{s, l}(t+\tau)\right] \\
& =\sigma_{g_{l}}^{2} J_{o}\left(2 \pi f_{m} \tau\right)
\end{aligned}
$$

where $J_{o}(\cdot)$ is the zeroth-order Bessel function of the first kind, and $f_{m}$ is the maximum Doppler spread, which is given by $V / \lambda$. $V$ and $\lambda$ are the mobile velocity and wavelength, respectively.

In $(2), I^{(d)}(t)$ is the overall interference due to thermal noise, multiple access interference, interchip interference, and some others and is modeled as zero-mean white Gaussian noise with two-side power spectral density of $I_{0} / 2(\mathrm{~W} / \mathrm{Hz})$ [22]. Using quadratic representation, then

$$
I^{(d)}(t)=\sqrt{2}\left[I_{c}^{(d)}(t) \cos \left(2 \pi f_{c} t\right)-I_{s}^{(d)}(t) \sin \left(2 \pi f_{c} t\right)\right]
$$

where $I_{c}^{(d)}(t)$ and $I_{s}^{(d)}(t)$ are also independent zero-mean white Gaussian processes with two-sided power spectral density $I_{0} / 2$ (W/Hz).

Two extreme types of channels will be investigated in the following. One is fast faded and the other is static. By fast faded, we mean that the channel varies fast enough so that each preamble transmission experiences independent fading. By the static channel, on the other hand, we mean that the channel is fixed during the entire burst synchronization process, and therefore, can be considered as an additive white Gaussian noise (AWGN) channel but with multiple rays. The results for other types of channels may be obtained with appropriate interpolation.

\section{BURST SYNCHRONIZERS}

The block diagram of the burst synchronizers is shown in Fig. 2. The received signal $r^{(d)}(t), d=1, \ldots, D$, after down conversion, chip-matched filtering, and sampling is detected by a parallel-serial code phase detector. The functionality of the detector is to produce the set of statistics $\left\{Z_{i}^{(d)}\right\}, i=1, \ldots, N_{u}$, which are combined to form

$$
Z_{i} \doteq \sum_{d=1}^{D} Z_{i}^{(d)}
$$

in order to obtain diversity reception. $Z_{i}$ is a measurement whether a preamble has arrived at the $i$ th cell $\mathcal{C}_{i}$ or not. Finally, the decision variable $Z$ is formed as

$$
Z \doteq \sum_{L_{c} \text { largest in }\left\{Z_{i}\right\}} Z_{i}
$$

and is used to test against a threshold $\eta_{0}$, where $1 \leq L_{c} \leq$ $L$ is the number of paths used for multipath combining. If the threshold is exceeded, then an ACK is sent back, otherwise, the test for a new preamble arrival continues. The consideration of using $L_{c}>1$ is to see if multipath combining is beneficial in the stage of burst synchronization. The constant false alarm rate (CFAR) will be used for setting the threshold $\eta_{0}$ in this study.

\section{A. Parallel-Serial Code Phase Detector}

Fig. 3 is the block diagram of the proposed parallel-serial code phase detector, where a total of $N \leq N_{u}$ active correlators are used for detecting $N_{u}$ code phases (cells). The correlation length of each correlator is set to $N$. The operation of this detector is best explained by using a simple example. Fig. 4 is such an example with $N_{u}=4, N=3$, and $K=11$, where $\nu_{n}$ is the input signal to a code-phase detector, a shaded block is to denote a length $N$ active correlation between $\left\{\nu_{n}\right\}$ and the corresponding spreading code $\left\{c_{n}^{*}\right\}$ (say for detecting $\mathcal{C}_{i}$ ), and $\# k$ in a block is to denote that the correlator $\# k$ is used for the correlation. The * denotes the complex conjugation. In addition, $y_{i}(j)$ is the $j$ th block correlation output for detecting the code phase $\mathcal{C}_{i}$.

As shown in Fig. 4, at first, the correlators (\#1, \#2, and \#3) are employed to detect the code phase $\mathcal{C}_{1}, \mathcal{C}_{2}$, and $\mathcal{C}_{3}$, respectively. Since the correlation length is equal to $N=3$, at the end of $n=2$ and after the output of $y_{1}(0)$, Correlator \#1 is free and can be used for the detection of $\mathcal{C}_{4}$. Likewise, at the end of $n=3$ and after the output of $y_{2}(0)$, Correlator \#2 is free and can be used for the detection of $\mathcal{C}_{1}$ again. In this way, $N$ correlators can be 


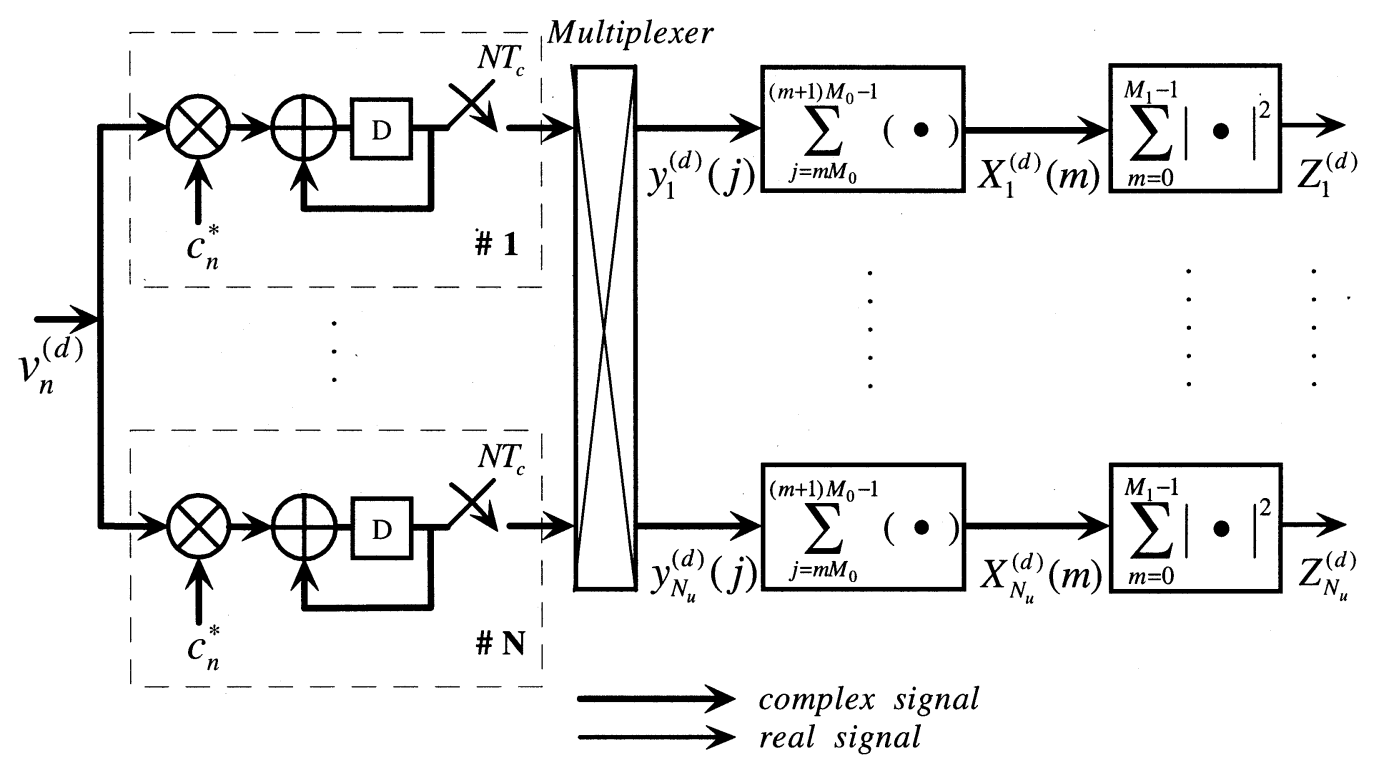

Fig. 3. Block diagram of the proposed parallel-serial code phase detector.

\begin{tabular}{|c|c|c|c|c|c|c|c|c|c|c|c|c|c|c|}
\hline $\mathrm{n}$ & 0 & 1 & 2 & 3 & 4 & 5 & 6 & 7 & 8 & 9 & 10 & 11 & 12 & 13 \\
\hline input sequence & $v_{0}$ & $v_{l}$ & $v_{2}$ & $v_{3}$ & $v_{4}$ & $v_{5}$ & $v_{6}$ & $v_{7}$ & $v_{8}$ & $v_{9}$ & $v_{10}$ & $v_{11}$ & $v_{12}$ & $v_{13}$ \\
\hline$C_{1}$ & ${ }^{\# 1} c_{0}{ }^{*}$ & $c_{1}^{*}$ & $c_{2}{ }^{*}$ & $c_{3}{ }^{*}$ & ${ }^{\# 2} c_{4}{ }^{*}$ & $c_{5}^{*}$ & $c_{6}{ }^{*}$ & $c_{7}{ }^{*}$ & ${ }^{\# 3} c_{8}{ }^{*}$ & $c_{9}{ }^{*}$ & $c_{10}{ }^{*}$ & & & \\
\hline$C_{2}$ & & ${ }^{\# 2} c_{0}^{*}$ & $c_{l}^{*}$ & $c_{2}{ }^{*}$ & $c_{3}{ }^{*}$ & ${ }^{\# 3} c_{4}{ }^{*}$ & $c_{5}^{*}$ & $c_{6}^{*}$ & $c_{7}^{*}$ & ${ }^{\# 1} c_{8}{ }^{*}$ & $c_{9}^{*}$ & $c_{10}{ }^{*}$ & & \\
\hline$C_{3}$ & & & ${ }^{\# 3} c_{0}{ }^{*}$ & $c_{I}^{*}$ & $c_{2}^{*}$ & $c_{3}{ }^{*}$ & $\# 1 c_{4}^{*}$ & $c_{5}^{*}$ & $c_{6}^{*}$ & $c_{7}{ }^{*}$ & ${ }^{\# 2} c_{8}{ }^{*}$ & $c_{9}{ }^{*}$ & $c_{10}{ }^{*}$ & \\
\hline$C_{4}$ & & & & ${ }^{\# 1} c_{0}{ }^{*}$ & $c_{I}^{*}$ & $c_{2}^{*}$ & $c_{3}{ }^{*}$ & ${ }^{\# 2} c_{4}{ }^{*}$ & $c_{5}^{*}$ & $c_{6}^{*}$ & $c_{7}^{*}$ & ${ }^{\# 3} c_{8}{ }^{*}$ & $c_{9}{ }^{*}$ & $c_{10}{ }^{*}$ \\
\hline output & & & $y_{1}(0)$ & $y_{2}(0)$ & $y_{3}(0)$ & $y_{4}(0)$ & $y_{1}(1)$ & $y_{2}(1)$ & $y_{3}(1)$ & $y_{4}(1)$ & $y_{1}(2)$ & $y_{2}(2)$ & $y_{3}(2)$ & $y_{4}(2)$ \\
\hline
\end{tabular}

Fig. 4. Simple example to illustrate the operation of the proposed parallel-serial code phase detector.

utilized fairly to detect the $N_{u}$ code phases. In Fig. 3, $M_{0}$ blocks of correlation output are coherently accumulated first and then accumulated noncoherently for $M_{1}$ times in order to alleviate the adverse effects of frequency offset and fast fading. It is clear that $M=M_{0} \cdot M_{1}$ is the total block of correlation involved in the detection.

Alternatively, the $N$ correlators in Fig. 3 can be replaced by an $N$-tap matched filter (passive) correlator [20]. To see this, again, use Fig. 4 as an example. Initially, the tap coefficients of the three-tap matched filter is downloaded with $c_{0}^{*}, c_{1}^{*}$, and $c_{2}^{*}$. Since $N=3, y_{1}(0), y_{2}(0), y_{3}(0)$, and $y_{4}(0)$ are available at the output of the matched filter at $n=2, n=3, n=4$, and $n=5$, respectively. (Note that at $n=2, \nu_{0}, \nu_{1}$, and $\nu_{2}$ resides in the matched filter, and at $n=3, \nu_{0}$ is kicked out while $\nu_{3}$ is pushed into the matched filter.) After obtaining $y_{4}(0)$, the tap coefficients are changed to $c_{4}^{*}, c_{5}^{*}$, and $c_{6}^{*}$. Then, $y_{1}(1)$ becomes available at $n=6, y_{2}(1)$ at $n=7$, and so forth. In fact, it was shown in [21] that the implementation with matched filter correlators is a bit simpler than with active correlators for WCDMA/FDD system under the same performance.

Several observations on the operation of the code-phase detector are worth mentioning here. First, since $N \leq N_{u}$, not all the received signal $\left\{\nu_{n}\right\}$ can be used in the detection, and that results in a performance degradation. For example, $\nu_{3}$ and $\nu_{7}$ in Fig. 4 are not used in the detection of $\mathcal{C}_{1}$. In addition, the gap between block correlations is equal to $N_{u}-N$ chips. Second, although not shown in Fig. 3, noncoherent accumulation may also be used in each block of $N$-chip correlation in order to alleviate the adverse effects of frequency offset and fast fading, depending on their sizes. Third, the structure is quite flexible in that both active and passive correlators can be employed and the parameters $N, M_{0}$, and $M_{1}$ can be easily selected to achieve different performance/complexity tradeoffs, as will be shown in Section V. And finally, depending on the code length $K$ of the preamble, the size of the last block of correlation may not be equal to $N$, and this needs to be taken care of in the performance analysis and implementation.

\section{Performance Analysis}

\section{A. Statistics of Variables $\left\{Z_{i}\right\}$}

The probability density function (pdf) and cumulative density function (cdf) of the variables $\left\{Z_{i}\right\}$ are derived here. Without loss of generality, the first $L$ code phases $\mathcal{C}_{i}, i=1 \ldots L$ will be assumed to be the $H_{1}$ cells, and all others are the $H_{0}$ cells. 
From Fig. 3, it can be shown [15], [22] that the output of the $j$ th block of correlation for the diversity branch $d$ is given as follows: ${ }^{3}$

$$
\begin{aligned}
y_{i}^{(d)}(j)=\sum_{n=0}^{N-1}[ & \sqrt{E_{c}^{(p)}} x_{i}^{(d)}\left(j N_{u}+n+i\right) \\
& \left.+I_{i}^{(d)}\left(j N_{u}+n+i\right)\right], \quad i=1 \ldots L
\end{aligned}
$$

and

$$
y_{i}^{(d)}(j)=\sum_{n=0}^{N-1} I_{i}^{(d)}\left(j N_{u}+n+i\right), \quad i=L+1 \ldots N_{u}
$$

where

$$
x_{i}^{(d)}(k) \doteq g_{i}^{(d)}(k) \exp \left[\boldsymbol{j} 2 \pi \Delta f k T_{c}\right]
$$

where $g_{i}^{(d)}(k) \doteq g_{i}^{(d)}\left(k T_{c}\right)$, and $\left\{I_{i}^{(d)}(n)\right\}$ are zero mean complex-valued Gaussian variables with independent real and imaginary parts and variance $1 / 2 \cdot E\left[\left|I_{i}^{(d)}(n)\right|^{2}\right]=I_{0} / 2$. Also, from Fig. 3

$$
\begin{aligned}
X_{i}^{(d)}(m) & =\sum_{j=0}^{M_{0}-1} y_{i}^{(d)}\left(m M_{0}+j\right), \quad m=0 \ldots M_{1}-1 \\
Z_{i}^{(d)} & =\sum_{m=0}^{M_{1}-1}\left|X_{i}^{(d)}(m)\right|^{2}
\end{aligned}
$$

and

$$
Z_{i}=\sum_{d=1}^{D} Z_{i}^{(d)}
$$

Define the complex random vector

$$
\boldsymbol{X}_{i}^{(d)} \doteq\left[X_{i}^{(d)}(0), X_{i}^{(d)}(1), \ldots, X_{i}^{(d)}\left(M_{1}-1\right)\right]^{T}
$$

where $T$ denotes the transpose operator. Then (12) becomes

$$
Z_{i}^{(d)}=\boldsymbol{X}_{i}^{(d) H} \boldsymbol{X}_{i}^{(d)}
$$

where $H$ denotes the Hermitian transpose.

1) $H_{0}$ Cells $\left(i=L+1 \ldots N_{u}\right)$ : From (9) and (11)-(13), $Z_{i}$ is a central chi-square distributed variable with $2 M_{1} D$ degrees of freedom. Thus, the pdf and cdf are given by [23]

$$
\begin{aligned}
f_{0}(z) & \doteq f_{Z_{i}}\left(z \mid H_{0}\right) \\
& =\frac{1}{\left(2 \sigma_{I}^{2}\right)^{M_{1} D}\left(M_{1} D-1\right) !} z^{M_{1} D-1} e^{-z / 2 \sigma_{I}^{2}} \\
F_{0}(z) & \doteq F_{Z_{i}}\left(z \mid H_{0}\right) \\
& =1-e^{-z / 2 \sigma_{I}^{2}} \sum_{k=0}^{M_{1} D-1} \frac{1}{k !}\left(\frac{z}{2 \sigma_{I}^{2}}\right)^{k}, \quad z \geq 0
\end{aligned}
$$

respectively, where $\sigma_{I}^{2}=M_{0} N\left(I_{0} / 2\right)$.

\footnotetext{
${ }^{3}$ For simplicity of presentation, an ideal chip sampling with no multipath interference is assumed. Note, however, that for small $M_{0} N$, the length of coherent accumulation, the multipath interference can be significant. As a result, $M_{0} N$ usually is taken large enough to have a satisfactory performance. In any case, as long as the multipath interference can be modeled as AWGN, the analysis given in the paper can be applied.
}

2) $H_{1}$ Cells $(i=1 \ldots L)$ : Two extreme types of channels will be investigated under $H_{1}$ cells. One is static and the other is fast faded. Recall that by static, we mean the channel remains unchanged during the entire burst synchronization process, and by fast fading, we mean the channel varies fast enough so that each preamble transmission experiences independent fading.

For the static channel, from (8) and (11)-(13), it is clear that $Z_{i}$ is a noncentral chi-square variable with $2 M_{1} D$ degrees of freedom. Therefore, from [23]

$$
\begin{aligned}
f_{1, i}(z) & \doteq f_{Z_{i}}\left(z \mid H_{1}\right) \\
& =\frac{1}{\left(2 \sigma_{I}^{2}\right)}\left(\frac{z}{s_{i}^{2}}\right)^{\frac{M_{1} D-1}{2}} e^{-\left(s_{i}^{2}+z\right) / 2 \sigma_{I}^{2}} \times I_{M_{1} D-1}\left(\frac{\sqrt{z} s_{i}}{\sigma_{I}^{2}}\right)
\end{aligned}
$$

and

$$
\begin{aligned}
F_{1, i}(z) & \doteq F_{Z_{i}}\left(z \mid H_{1}\right) \\
& =1-Q_{M_{1} D}\left(\frac{s_{i}}{\sigma_{I}}, \frac{\sqrt{z}}{\sigma_{I}}\right), \quad z \geq 0
\end{aligned}
$$

where $I_{\alpha}(x)$ is the $\alpha$ th-order modified Bessel function of the first kind, $Q_{\beta}(a, b)$ is the generalized Marcum $Q$ function with order $\beta$, and $s_{i}^{2}$ is the noncentrality. From Appendix A, $s_{i}^{2}$ is derived as

$$
\begin{aligned}
& s_{i}^{2}=E_{c}^{(p)}\left|g_{i}\right|^{2} M_{1} D N^{2} M_{0}^{2} \cdot \operatorname{sinc}^{2}\left(N \Delta f T_{c}\right) \\
& \cdot \operatorname{sinc}^{2}\left(M_{0} N_{u} \Delta f T_{c}\right) .
\end{aligned}
$$

For the fast-faded channel, the pdf and cdf can be obtained as follows. Since (12) is a quadratic form and $\left\{Z_{i}^{(d)}\right\}$ are i.i.d. variables, the moment generating function of $Z_{i}$ is given as [24]

$$
\Phi_{i}(s)=\prod_{m=0}^{M_{1-1}}\left(1+s \lambda_{i, m}\right)^{-D}, \quad i=1 \ldots L
$$

where $\left\{\lambda_{i, m}\right\}$ are the eigenvalues of the correlation matrix

$$
R_{X_{i}}=R_{X_{i}}^{(d)} \doteq E\left[\boldsymbol{X}_{i}^{(d)} \boldsymbol{X}_{i}^{(d) H}\right], \quad d=1 \ldots D
$$

and $R_{X_{i}}$ is given in Appendix B.

In order to avoid a numerical problem that is usually encountered by using inverse Laplace method to find the pdf of $Z_{i}$, as did in [15], a saddle-point integration technique [25] is employed here instead. As shown in Appendix C, the pdf and cdf of $Z_{i}$ can be evaluated as

$$
\begin{aligned}
f_{1, i}(z)= & \frac{e^{z s_{0}}}{\pi} \int_{0}^{\infty} A_{i}\left(s_{0}, y\right) \cos \left[\Theta_{i}\left(s_{0}, y\right)+z y\right] d y \\
F_{1, i}(z)= & \frac{e^{z s_{0}}}{\pi} \int_{0}^{\infty} A_{i}\left(s_{0}, y\right)\left(s_{0}^{2}+y^{2}\right)^{-\frac{1}{2}} \\
& \times \sin \left[\Theta_{i}\left(s_{0}, y\right)+z y+\tan ^{-1} \frac{s_{0}}{y}\right] d y
\end{aligned}
$$


respectively, where $s_{0}$ is the saddle point of the function $H_{i}(s)=\Phi_{i}(s) e^{z s} / s$

$$
A_{i}\left(s_{0}, y\right)=\prod_{m=0}^{M_{1}-1}\left[\left(1+s_{0} \lambda_{i, m}\right)^{2}+\left(y \lambda_{i, m}\right)^{2}\right]^{-\frac{D}{2}}
$$

and

$$
\Theta_{i}\left(s_{0}, y\right)=D \cdot \sum_{m=0}^{M_{1}-1} \tan ^{-1}\left(\frac{-y \lambda_{i, m}}{1+s_{0} \lambda_{i, m}}\right) .
$$

Equations (23) and (22) can be evaluated numerically, as discussed in Appendix C.

\section{B. Performance Measures}

Probability of detection $P_{D}$ (for single preamble), probability of synchronization $P_{\mathrm{SYN}}$, mean ramping number $N_{\text {ramp }}$, and average transmit chip energy $E_{c, \text { avg }}$ are performance measures used to characterize the synchronizer performance. To analyze, however, it is helpful to define the probabilities of detection, miss, and false detection for the detection of each individual preamble first. These probabilities are defined as follows for the detection of the $p$ th preamble.

$P_{D}^{(p)} \doteq \operatorname{Pr}\left\{Z \geq \eta_{0}\right.$ and the selected $\mathcal{C}_{i}$ is one of the $H_{1}$ cells $\mid$ The $p$ th preamble is present. $\}$;

$P_{M}^{(p)} \doteq \operatorname{Pr}\left\{Z<\eta_{0} \mid\right.$ The $p$ th preamble is present. $\}$;

$P_{\mathrm{FD}}^{(p)} \doteq \operatorname{Pr}\left\{Z \geq \eta_{0}\right.$ and the selected $\mathcal{C}_{i}$ is not an $H_{1}$ cell $\mid$ The $p$ th preamble is present. $\}$.

It is evident that $P_{D}^{(p)}+P_{M}^{(p)}+P_{\mathrm{FD}}^{(p)}=1$. Furthermore, if a false detection happens, the synchronization process will be stopped, and the attempt is considered to be a failure.

For the considered channels, preamble detections are independent of each other. Therefore, the probability that the burst is synchronized at the $p$ th preamble $\left(P_{\mathrm{SYN}}^{(p)}\right)$ is given by

$$
P_{\mathrm{SYN}}^{(p)}=P_{D}^{(p)} \cdot \prod_{k=0}^{p-1} P_{M}^{(k)}, \quad 1 \leq p \leq N_{P}
$$

the average chip energy is

$$
E_{c, \mathrm{avg}}=\sum_{p=1}^{N_{P}}\left[E_{c}^{(p)} \cdot \prod_{k=0}^{p-1} P_{M}^{(k)}\right]
$$

and the mean ramping number is

$$
N_{\text {ramp }}=\sum_{p=1}^{N_{P}}\left[\prod_{k=0}^{p-1} P_{M}^{(k)}\right]
$$

where $P_{M}^{(0)}=1$. Taking the power control error into account, (26)-(28) are then modified as

$$
\begin{aligned}
P_{\mathrm{SYN}}^{(p)} & =E\left[P_{\mathrm{SYN}}^{(p)}(\Psi)\right] \\
E_{c, \text { avg }} & =E\left[E_{c, \operatorname{avg}}(\Psi)\right]
\end{aligned}
$$

and

$$
N_{\text {ramp }}=E\left[N_{\text {ramp }}(\Psi)\right]
$$

where $\Psi$ is a truncated log-normal variable used to characterize the power control error. In this study, $\Psi$ will be truncated at the $k$ times of standard deviation $\sigma_{\Psi}$, where $k$ is a positive integer.

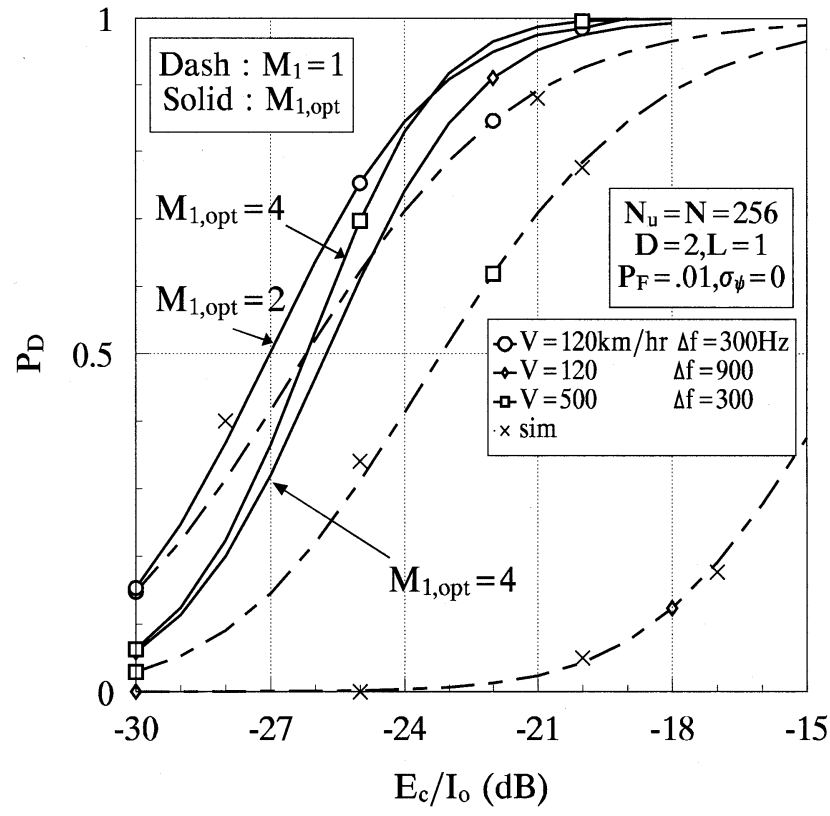

Fig. 5. Effects of noncoherent accumulation on the detection probability for different fading rates and frequency offsets.

Finally, the synchronization probability of the synchronizer is given by

$$
P_{\mathrm{SYN}}=\sum_{p=1}^{N_{P}} P_{\mathrm{SYN}}^{(p)} \cdot
$$

In Fig. 2, $\eta_{0}$ will be selected according to a constant false alarm rate, which is defined as

$$
P_{F} \doteq \operatorname{Pr}\left\{Z \geq \eta_{0} \mid \text { Preamble is not present. }\right\} \text {. }
$$

$P_{D}^{(p)}, P_{M}^{(p)}, P_{\mathrm{FD}}^{(p)}$, and $P_{F}$ are evaluated in Appendix D for the cases with or without multipath combining.

\section{NUMERICAL RESUlTS}

System parameters that resembles those used in the 3GPP WCDMA/FDD system are adopted here for numerical examples. One of the preamble spreading codes specified in the 3GPP WCDMA/FDD system is employed [1]. The chip rates is $3.84 \mathrm{M}$ chips/s, the preamble length is 4096 chips, and the carrier frequency is $2 \mathrm{GHz}$. Four different cell radii are considered, that is $1.25,5,10$, and $20 \mathrm{~km}$ which corresponds to the time uncertainty of $N_{u}=32,128,256$, and 512 chips, respectively. In addition, $M_{0}=1$ is used for all the numerical results. In the simulation, Jake's simulation model [19] is employed for fast-faded channels. Nonoverlapped segments of the simulator output are used to generate independent fading, and 10000 access attempts have been simulated to obtain accurate simulation results.

Fig. 5 shows the effect of frequency offset and fading rates on the detection probability. As expected, $M_{1}$ needs to be chosen properly in order to obtain better performance. The optimum $M_{1}$ varies with the sizes of frequency offset and fading rate. As shown, a vehicular speed of $120 \mathrm{~km}$ combined with $900-\mathrm{Hz}$ frequency offset happens to give a very bad performance, if $M_{1}=1$ is used. The $900-\mathrm{Hz}$ frequency offset corresponds to 


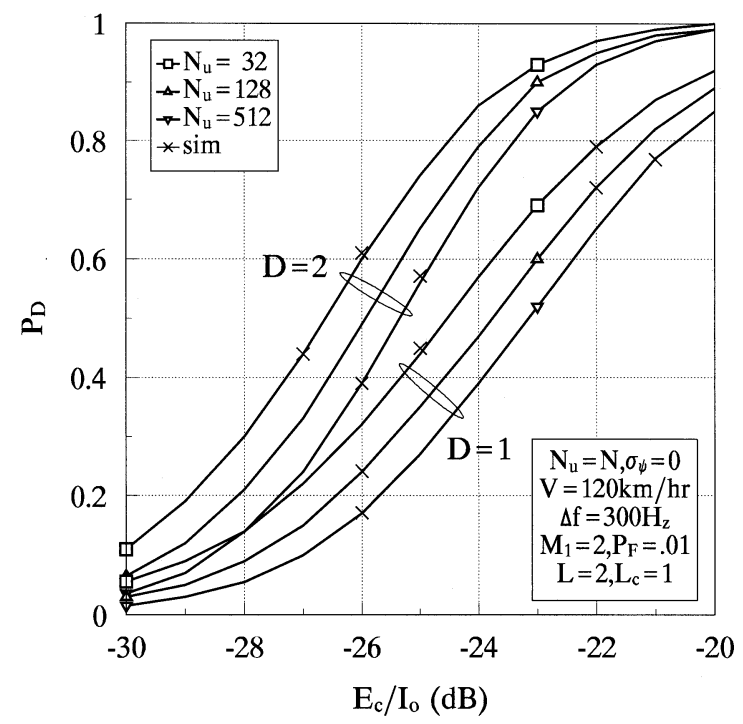

Fig. 6. Comparisons of detection probability under different time uncertainty with and without diversity reception.

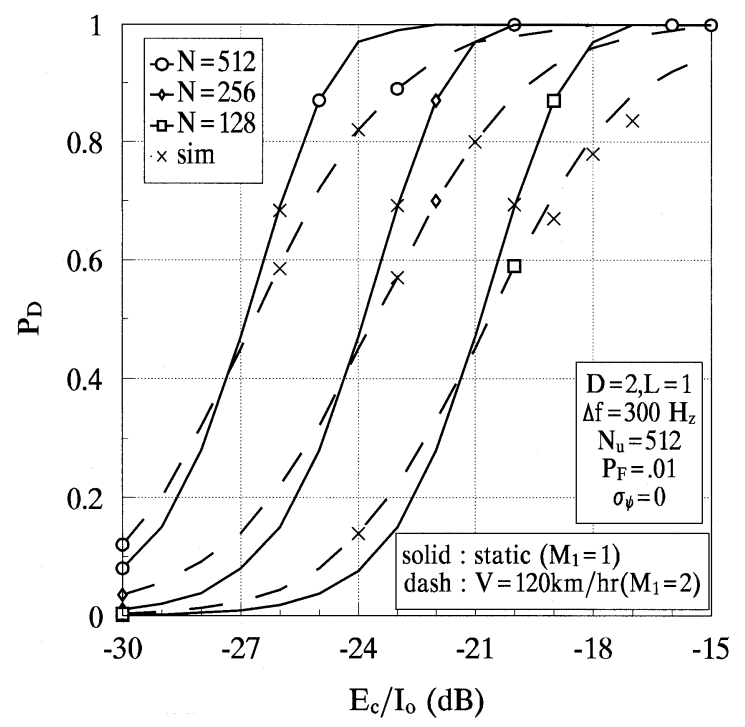

Fig. 7. Performance and complexity tradeoffs with different number of correlators.

$0.45 \mathrm{ppm}$ in oscillator stability. Fig. 5 also shows a close match between analytical and simulation results, and that implies that the effect of the correlations between different preamble detections is negligible. Recall that in the analysis, we assume that preamble detections are independent of each other. In the figure, $\sigma_{\psi}=0$ means that there is no power control error.

In Fig. 6, the detection probability is investigated with different $N_{u}$ and with or without diversity reception. As shown, 2to 3-dB improvement is observed with $D=2$ for the signal-tonoise ratio of interests, and a large $N_{u}$ results in a performance degradation. This is attributed to the fact that for a large $N_{u}$, a larger $\eta_{0}$ is necessary to have a CFAR, and that decreases the detection probability. Fig. 7 compares the detection probability of using different number of correlators $N$. Basically, a 3-dB performance loss is observed when $N$ is reduced by half for both fading and static channels.

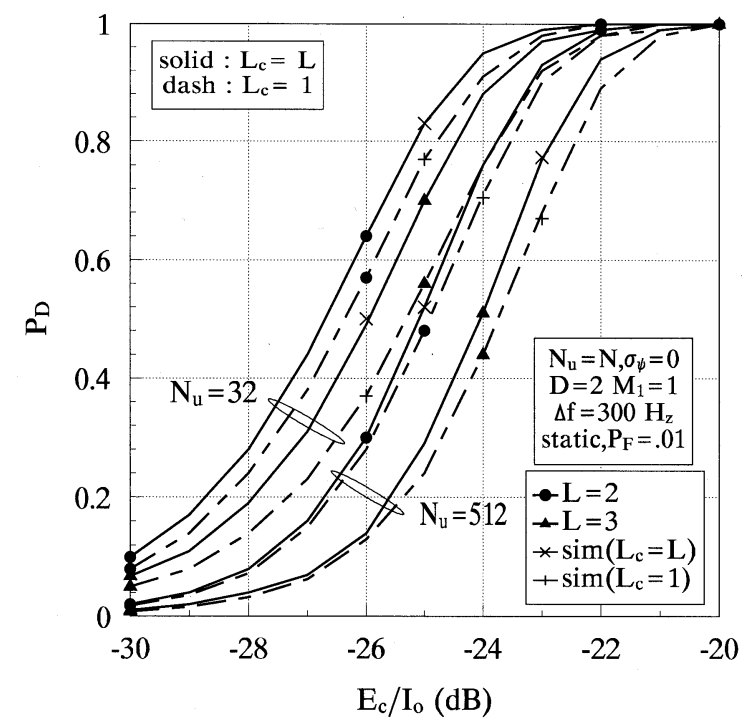

Fig. 8. Performance gains with multipath combining for static channels with equal path power.

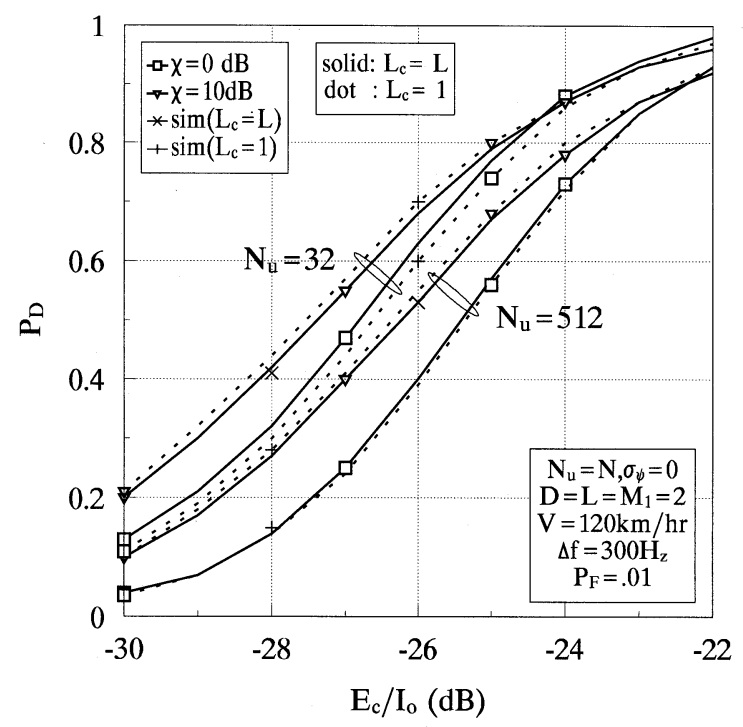

Fig. 9. Performance gains with multipath combining for fading channels with unequal path power.

In Figs. 8 and 9, the effects of multipath combining are investigated. As shown in Fig. 8, a gain of a fraction of decibels is obtained by using multipath combining for two or three paths with equal power. Nevertheless, the gain becomes diminishing or even becomes negative when the paths have different power as observed in Fig. 9, where $\chi(\mathrm{dB})$ is the power ratio of the first to the second path.

Fig. 10 shows the interrelations among $P_{\mathrm{SYN}}, N_{\text {ramp }}$, and $N_{P}$ with $P_{F}$ as a parameter. As can be seen, a small $P_{F}$ along with a large $N_{P}$ is a necessity for having a high $P_{\mathrm{SYN}}$. Of course, the paid price is a larger $N_{\text {ramp }}$. Furthermore, depending on the size $P_{F}, P_{\mathrm{SYN}}$ becomes saturated no matter how large $N_{P}$ is used, due to a nonzero $P_{\mathrm{FD}}$. Similarly, Fig. 11 illustrates the same relationships but with power control error as a parameter. As expected, a large power control error results in a significant performance loss in terms of $P_{\mathrm{SYN}}$ and $N_{\text {ramp. }}$. 


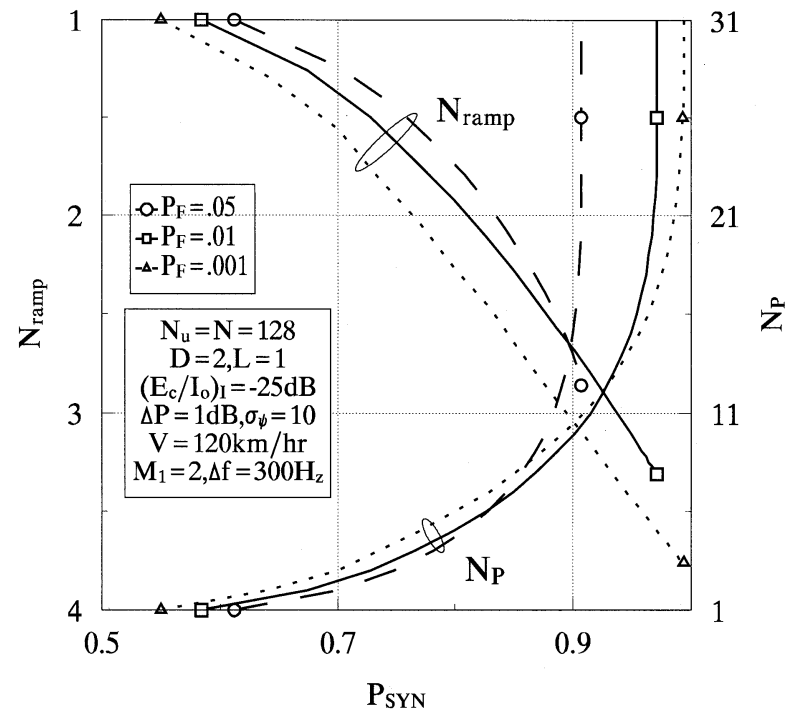

Fig. 10. Selection of $\eta_{0}$ and its effects on synchronizer's performance.

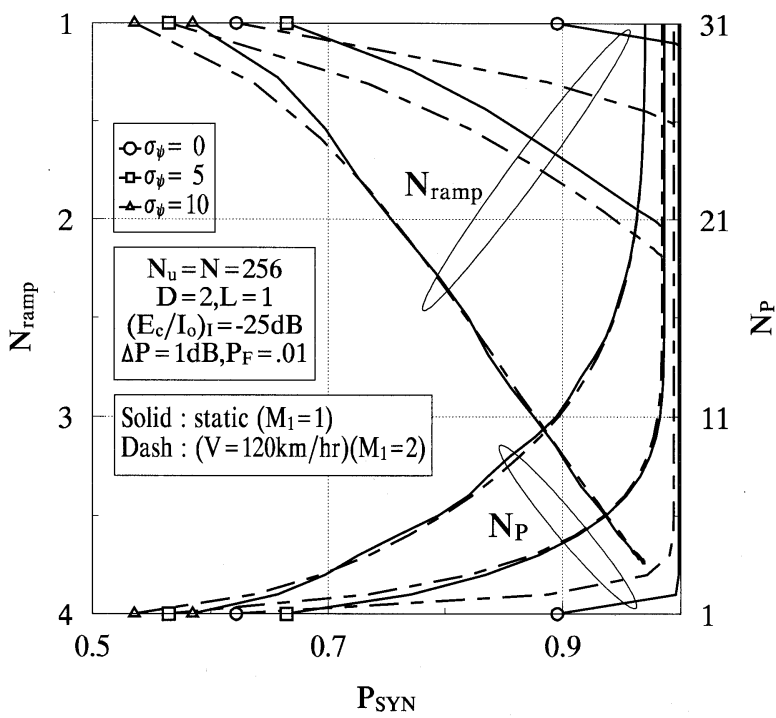

Fig. 11. Effects of power control error on the synchronizer's performance.

Fig. 12 shows a typical example that the ramping step affects the synchronizer's performance. As seen, a smaller ramping number can achieve the same level of $P_{\mathrm{SYN}}$, when a large ramping step is used, only at an expense of a slightly larger averaged transmit power (energy). In addition, under a CFAR, a larger attainable $P_{\text {SYN }}$ can always be obtained with a large ramping step. Nevertheless, one drawback with a large ramping step is that the transmit power of each preamble is larger than that with a smaller one, because the total power needs to be transmitted in a fewer preambles, and this might not be desirable to the operation of CDMA systems. In Fig. 13, typical effects of power control error on the performance of $P_{\mathrm{SYN}}, N_{\mathrm{ramp}}$ and the average transmit power are illustrated, where $\Psi\left(k \sigma_{\Psi}\right)$ is to denote that the log-normal variable $\Psi$ is truncated at $\pm k \sigma_{\Psi}$. As expected, a smaller power control error

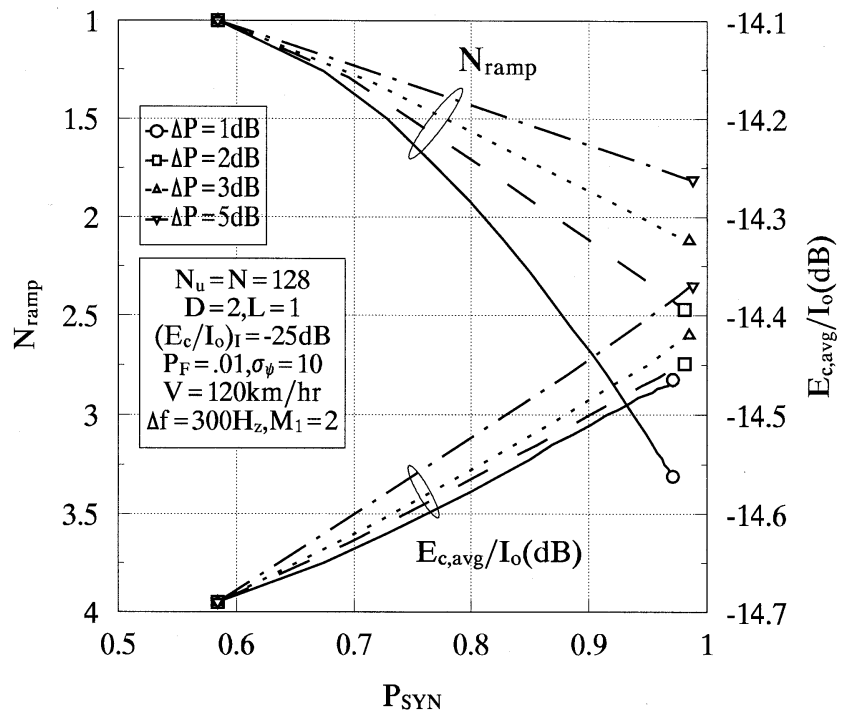

Fig. 12. Selection of ramping steps and its effects on the synchronizer's performance

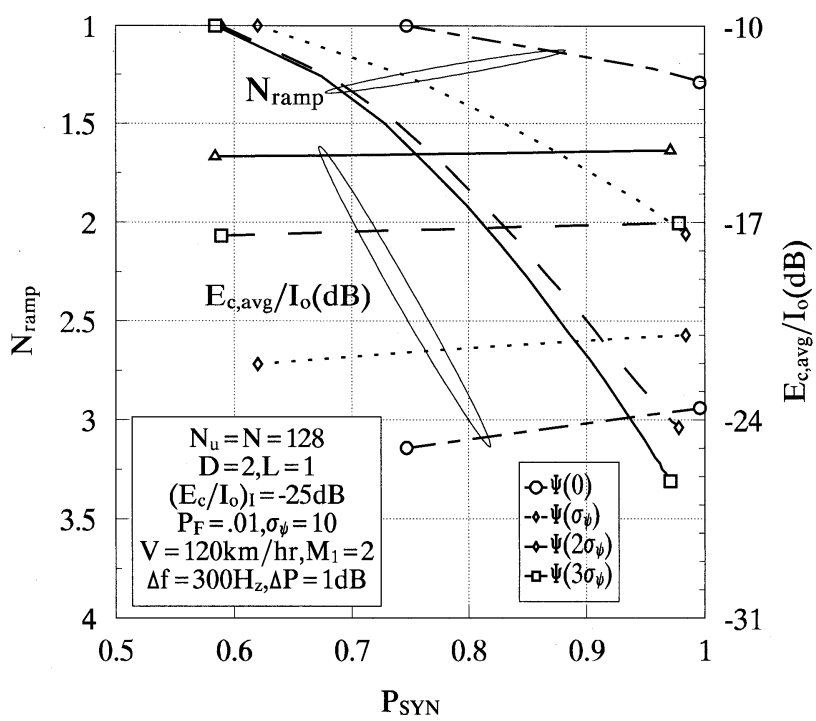

Fig. 13. Effects of power control error on the synchronizer's performance.

results in a large attainable $P_{\mathrm{SYN}}$, and a smaller $N_{\text {ramp }}$ and averaged transmit power.

\section{CONCLUSION}

In this paper, a burst synchronizer based on a flexible parallel-serial code phase detector has been proposed for random access with preamble power ramping in the reverse link of CDMA systems. A thorough analysis has been conducted with various system parameters being taken into account, including the number of correlators, nocoherent accumulation length, power control error, ramping step, diversity order, fading and frequency offsets, multipath combining, and others. Numerical results show that the synchronizer can be configured easily to achieve different performance/complexity tradeoffs, by selecting suitable system parameters. 
APPENDIX A

EVALUATION OF THE NONCENTRALITY $s_{i}^{2}$ FOR STATIC CHANNELS

From definition, the noncentrality $s_{i}^{2}$ for the variable $Z_{i}$ is given by

$$
\begin{aligned}
s_{i}^{2} & \doteq D \cdot \sum_{m=0}^{M_{1}-1}\left|E\left[X_{i}(m)\right]\right|^{2} \\
& =D \cdot \sum_{m=0}^{M_{1}-1}\left[E^{2}\left[X_{i, c}(m)\right]+E^{2}\left[X_{i, s}(m)\right]\right]
\end{aligned}
$$

where $X_{i}(m) \doteq X_{i, c}(m)+\boldsymbol{j} X_{i, s}(m)$. From (8), (10), and (11), we have

$$
\begin{aligned}
& E\left[X_{i, c}(m)\right] \\
& \quad=\sum_{j=0}^{M_{0}-1} \sum_{n=0}^{N-1} \sqrt{E_{c}^{(p)}}\left|g_{i}\right| \\
& \quad \times \cos \left[2 \pi \Delta f T_{c}\left(\left(m M_{0}+j\right) N_{u}+n+i\right)+\theta_{i}\right]
\end{aligned}
$$

and

$$
\begin{aligned}
& E\left[X_{i, s}(m)\right] \\
& =\sum_{j=0}^{M_{0}-1} \sum_{n=0}^{N-1} \sqrt{E_{c}^{(p)}}\left|g_{i}\right| \\
& \times \sin \left[2 \pi \Delta f T_{c}\left(\left(m M_{0}+j\right) N_{u}+n+i\right)+\theta_{i}\right]
\end{aligned}
$$

where $g_{i} \doteq\left|g_{i}\right| \exp \left[\boldsymbol{j} \theta_{i}\right]$. By using

$$
\begin{aligned}
& \sum_{n=0}^{N-1} \cos (x+n y) \\
& \quad=\cos \left(x+\frac{N-1}{2} y\right) \sin \left(\frac{N y}{2}\right) \csc \left(\frac{y}{2}\right) \\
& \sum_{n=0}^{N-1} \sin (x+n y) \\
& \quad=\sin \left(x+\frac{N-1}{2} y\right) \sin \left(\frac{N y}{2}\right) \csc \left(\frac{y}{2}\right)
\end{aligned}
$$

along with

$$
\lim _{\Delta f T_{c} \rightarrow 0} \frac{1}{N} \sin \left(\pi N \Delta f T_{c}\right) \csc \left(\pi \Delta f T_{c}\right)=\operatorname{sinc}\left(N \Delta f T_{c}\right)
$$

where $s_{i}^{2}$ is obtained as

$$
\begin{aligned}
& s_{i}^{2}=D E_{c}^{(p)}\left|g_{i}\right|^{2} M_{1} N^{2} M_{0}^{2} \cdot \operatorname{sinc}^{2}\left(N \Delta f T_{c}\right) \\
& \cdot \operatorname{sinc}^{2}\left(M_{0} N_{u} \Delta f T_{c}\right) .
\end{aligned}
$$

\section{APPENDIX B}

CORRElation MATRIX $R_{X_{i}}$ FOR FAST-FADED Channels

From $(21)$, the $(m, n)$ th element of the correlation matrix $R_{X_{i}}$ is given by

$$
\begin{aligned}
{\left[R_{X_{i}}\right]_{m, n} } & \\
\doteq & E\left[X_{i}(m) X_{i}^{*}(n)\right] \\
= & E\left[X_{i, c}(m) X_{i, c}(n)\right]+E\left[X_{i, s}(m) X_{i, s}(n)\right] \\
& +\boldsymbol{j}\left[E\left[X_{i, s}(m) X_{i, c}(n)\right]-E\left[X_{i, c}(m) X_{i, s}(n)\right]\right]
\end{aligned}
$$

where

$$
\begin{aligned}
& X_{i, c}(k)=\sum_{j=0}^{M_{0}-1} \sum_{n=0}^{N-1}\left[\sqrt{E_{c}^{(p)}} x_{i, c}\left(\left(k M_{0}+j\right) N_{u}+n+i\right)\right. \\
& \left.+I_{i, c}\left(\left(k M_{0}+j\right) N_{u}+n+i\right)\right] \\
& X_{i, s}(k)=\sum_{j=0}^{M_{0}-1} \sum_{n=0}^{N-1}\left[\sqrt{E_{c}^{(p)}} x_{i, s}\left(\left(k M_{0}+j\right) N_{u}+n+i\right)\right. \\
& \left.+I_{i, s}\left(\left(k M_{0}+j\right) N_{u}+n+i\right)\right]
\end{aligned}
$$

with

$$
\begin{aligned}
x_{i, c}(k) \doteq & \operatorname{Re}\left[x_{i}(k)\right] \\
= & g_{i, c}(k) \cos \left(2 \pi \Delta f k T_{c}+\theta_{i}\right) \\
& -g_{i, s}(k) \sin \left(2 \pi \Delta f k T_{c}+\theta_{i}\right)
\end{aligned}
$$

and

$$
\begin{aligned}
x_{i, s}(k) \doteq & \operatorname{Im}\left[x_{i}(k)\right] \\
= & g_{i, c}(k) \sin \left(2 \pi \Delta f k T_{c}+\theta_{i}\right) \\
& +g_{i, s}(k) \cos \left(2 \pi \Delta f k T_{c}+\theta_{i}\right) .
\end{aligned}
$$

It is easy to show that

$$
\begin{aligned}
E\left[x_{i, c}(k) x_{i, c}(k+l)\right] & =E\left[x_{i, s}(k) x_{i, s}(k+l)\right] \\
& =R_{g_{i}}\left(l T_{c}\right) \cos \left(2 \pi \Delta f l T_{c}\right)
\end{aligned}
$$

and

$$
\begin{aligned}
E\left[x_{i, c}(k) x_{i, s}(k+l)\right] & =-E\left[x_{i, s}(k) x_{i, c}(k+l)\right] \\
& =R_{g_{i}}\left(l T_{c}\right) \sin \left(2 \pi \Delta f l T_{c}\right) .
\end{aligned}
$$

In addition, after some algebraic manipulation, we have

$$
\begin{aligned}
{\left[R_{X_{i}}\right]_{m, n}=} & \sum_{j_{1}=0}^{M_{0}-1} \sum_{j_{2}=0}^{M_{0}-1} \sum_{n_{1}=0}^{N-1} \sum_{n_{2}=0}^{N-1} 2 E_{c}^{(p)} R_{g_{i}} \\
& \cdot\left[\left(\left(k\left(m, j_{1}, n_{1}\right)-k\left(n, j_{2}, n_{2}\right)\right) T_{c}\right]\right. \\
& \cdot \exp \left[-j 2 \pi \Delta f T_{c}\left(k\left(m, j_{1}, n_{1}\right)-k\left(n, j_{2}, n_{2}\right)\right)\right] \\
& +N M_{0} \frac{I_{o}}{2} \delta_{m n}
\end{aligned}
$$

where $k(m, j, n) \doteq\left(m M_{0}+j\right) N_{u}+n$, and $\delta_{m n}$ is the Kronecker delta function, defined by

$$
\delta_{m n} \doteq\left\{\begin{array}{ll}
1, & \text { if } m=n \\
0, & \text { if } m \neq n
\end{array} .\right.
$$

\section{APPENDIX C}

\section{EVALUATION OF $F_{1, i}(z)$ AND $f_{1, i}(z)$ With SADDLE-POINT INTEGRATION}

In this appendix, we outline the procedure of using saddlepoint integration for the evaluation of $F_{1, i}(z)$ and $f_{1, i}(z)$ under $H_{1}$, that is $i=1, \ldots, L$. From [25], $F_{1, i}(z)$ can be evaluated efficiently by

$$
\begin{aligned}
F_{1, i}(z) & \doteq \int_{-\infty}^{z} f_{1, i}(x) d x \\
& =\frac{1}{\pi} \int_{0}^{\infty} \operatorname{Re}\left[H_{i}\left(s_{0}+\boldsymbol{j} y\right)\right] d y
\end{aligned}
$$


where $H_{i}(s) \doteq \Phi_{i}(s) e^{s z} / s$, and $s_{0}$ is the saddle point of $H_{i}(s)$. In other words, $d H_{i}(s) /\left.d s\right|_{s=s_{0}}=0$. Recall that

$$
\Phi_{i}(s)=\prod_{m=0}^{M_{1}-1}\left[\left(1+s \lambda_{i, m}\right)\right]^{-D}
$$

is the moment generating function of $Z_{i}$.

In our case, after some algebraic manipulation, it can be shown that

$\operatorname{Re}\left[H_{i}(x+\boldsymbol{j} y)\right]$

$$
=\frac{A_{i}(x, y) e^{z x}}{\sqrt{x^{2}+y^{2}}} \sin \left(\Theta_{i}(x, y)+z y+\tan ^{-1} \frac{x}{y}\right)
$$

where

$$
\begin{aligned}
& A_{i}(x, y)=\prod_{m=0}^{M_{1}-1}\left[\left(1+x \lambda_{i, m}\right)^{2}+\left(y \lambda_{i, m}\right)^{2}\right]^{-\frac{D}{2}} \\
& \Theta_{i}(x, y)=D \cdot \sum_{m=0}^{M_{1}-1} \tan ^{-1}\left(\frac{-y \lambda_{i, m}}{1+x \lambda_{i, m}}\right) .
\end{aligned}
$$

Substitute (49) into (48), we obtain

and

$$
\begin{aligned}
F_{1, i}(z)= & \frac{e^{z s_{0}}}{\pi} \int_{0}^{\infty} A_{i}\left(s_{0}, y\right)\left(s_{0}^{2}+y^{2}\right)^{-\frac{1}{2}} \\
& \times \sin \left[\Theta_{i}\left(s_{0}, y\right)+z y+\tan ^{-1} \frac{s_{0}}{y}\right] d y
\end{aligned}
$$

$$
\begin{aligned}
f_{1, i}(z) & \doteq \frac{d}{d z} F_{1, i}(z) \\
& =\frac{e^{z s_{0}}}{\pi} \int_{0}^{\infty} A_{i}\left(s_{0}, y\right) \cos \left[\Theta_{i}\left(s_{0}, y\right)+z y\right] d y .
\end{aligned}
$$

In addition

$$
\begin{aligned}
\frac{d}{d x} H_{i}(x)=\frac{e^{z x}}{x^{2}} \cdot\left[\prod_{m=0}^{M_{1}-1}\left(1+x \lambda_{i, m}\right)\right]^{-D} & \\
& \cdot\left(z x-1-\sum_{m=0}^{M_{1}-1} \frac{x D \lambda_{i, m}}{1+x \lambda_{i, m}}\right)
\end{aligned}
$$

and the saddle point $s_{0}$ can be solved easily by Newton's method or secant method [26].

In practice, the integrals in (52) and (53) can be evaluated numerically by the trapezoidal rule which approximates a typical semiinfinite integral by

$$
I=\int_{0}^{\infty} t(y) d y \cong \delta y \cdot\left[\frac{1}{2} t(0)+\sum_{k=1}^{\mathcal{K}} t(k \delta y)\right] .
$$

The approximation of (55) can be made as accurate as desirable by using a large $\mathcal{K}$ and a small $\delta y$, respectively. As in [25], $\delta y$ can be initially taken as

$$
\delta y=\left[\frac{d^{2}}{d s^{2}} \ln H_{i}\left(s_{0}\right)\right]^{-1 / 2}
$$

and then halved successively until the desired precision is obtained.

\section{APPENDIX D \\ Evaluation of $P_{F}, P_{D}, P_{\mathrm{FD}}$, AND $P_{M}$}

In this Appendix, the probabilities of false alarm $P_{F}$, detection $P_{D}^{(p)}$, false detection $P_{\mathrm{FD}}^{(p)}$, and miss $P_{M}^{(p)}$ for the $p$ th preamble are derived. Recall that

$P_{F} \doteq \operatorname{Pr}\left\{Z \geq \eta_{0} \mid\right.$ Preamble is not present. $\}$;

$P_{D}^{(p)} \doteq \operatorname{Pr}\left\{Z \geq \eta_{0}\right.$ and the selected $\mathcal{C}_{i}$ is one of the $H_{1}$ cells $\mid$ The $p$ th preamble is present. $\}$;

$P_{M}^{(p)} \doteq \operatorname{Pr}\left\{Z<\eta_{o} \mid\right.$ The $p$ th preamble is present. $\}$;

$P_{\mathrm{FD}}^{(p)} \doteq \operatorname{Pr}\left\{Z \geq \eta_{0}\right.$ and the selected $\mathcal{C}_{i}$ is not a $H_{1}$ cell $\mid$

The $p$ th preamble is present $\}$.

For notation simplicity, the superscript $(p)$ will be omitted in the following.

From (7), the decision variable $Z$ is defined as

$$
Z \doteq \sum_{L_{c} \text { largest in }\left\{Z_{i}\right\}} Z_{i} \text {. }
$$

Only the case of $L_{c}=L=2$ will be used as an example for illustrating the derivation. Using the same idea, similar but much more complicated expressions can be obtained for the cases of $L_{c}>2$.

From definition, it can be seen that

$$
P_{F}=\iint_{\Gamma_{2}}\left[\begin{array}{c}
N_{u}-2 \\
2
\end{array}\right] F_{0}^{N_{u}-2}\left(x_{2}\right) f_{0}\left(x_{2}\right) f_{0}\left(x_{1}\right) d x_{2} d x_{1}
$$

where $\left[\begin{array}{l}n \\ r\end{array}\right] \doteq(n ! /(n-r) !), f_{0}(x)$ and $F_{0}(x)$ are the pdf and cdf of $Z_{i}$ under $H_{0}$, respectively, and

$$
\begin{aligned}
\Gamma_{2} & =\left\{\left(x_{1}, x_{2}\right) \mid 0 \leq x_{2} \leq x_{1}, x_{1}+x_{2} \geq \eta_{0}\right\} \\
& =\left\{\left(x_{1}, x_{2}\right) \mid \eta_{0} / 2 \leq x_{1}, \max \left(0, \eta_{o}-x_{1}\right) \leq x_{2} \leq x_{1}\right\} .
\end{aligned}
$$

In addition

$$
\begin{aligned}
P_{D}= & \iint_{\Gamma_{2}} F_{0}^{N_{u}-2}\left(x_{2}\right)\left[f_{1,1}\left(x_{2}\right) f_{1,2}\left(x_{1}\right)\right. \\
& \left.+f_{1,2}\left(x_{2}\right) f_{1,1}\left(x_{1}\right)\right] d x_{2} d x_{1} \\
& +\iint_{\Gamma_{2}}\left(N_{u}-2\right) F_{0}^{N_{u}-3}\left(x_{2}\right) \\
& \times F_{1,1}\left(x_{2}\right)\left[f_{1,2}\left(x_{2}\right) f_{0}\left(x_{1}\right)+f_{0}\left(x_{2}\right) f_{1,2}\left(x_{1}\right)\right] d x_{2} d x_{1} \\
& +\iint_{\Gamma_{2}}\left(N_{u}-2\right) F_{0}^{N_{u}-3}\left(x_{2}\right) F_{1,2}\left(x_{2}\right) \\
& \left.\times\left[f_{1,1}\left(x_{2}\right) f_{0}\left(x_{1}\right)+f_{0}\left(x_{2}\right) f_{1,1}\left(x_{1}\right)\right]\right\} d x_{2} d x_{1}
\end{aligned}
$$

where $f_{1, i}(x)$ and $F_{1, i}(x), i=1,2$ are the pdf and cdf of the $Z_{i}$ under the $i$ th $H_{1}$ cell, respectively. In the above equation, the first term accounts for the detection probability of both the $H_{1}$ cells used for combining, and the second and third terms account for the cases with only one $H_{1}$ cell being used in the combining. Finally

$$
\begin{array}{r}
P_{\mathrm{FD}}=\iint_{\Gamma_{2}}\left[\begin{array}{c}
N_{u}-2 \\
2
\end{array}\right] F_{0}^{N_{u}-4}\left(x_{2}\right) F_{1,1}\left(x_{2}\right) F_{1,2}\left(x_{2}\right) \\
\times f_{0}\left(x_{2}\right) f_{0}\left(x_{1}\right) d x_{2} d x_{1}
\end{array}
$$

and $P_{M}=1-P_{D}-P_{\mathrm{FD}}$. 


\section{REFERENCES}

[1] 3rd Generation Partnership Project; Technical Specification Group Radio Access Network, 3G TS 25.211-215, v3.5.0, Mar. 2001.

[2] Physical Layer Standard for CDMA 2000 Spread Spectrum Systems, TIA/EIA Interim Standard, Aug. 1999.

[3] S. S. Lim, Q. Cao, C. Demeterscu, D. J. Reader, and J. Lin, "3rd generation RACH transmission-A candidate," in Proc. IEEE VTC'99, Houston, TX, May 1999, pp. 140-144.

[4] R. Esmailzadeh and M. Gustafsson, "A new slotted Aloha based random access method for CDMA systems," in Proc. IEEE ICUPC'97, San Diego, CA, Oct. 1997, pp. 43-47.

[5] "PAPR Reduction Scheme for RACH Preamble," Ericsson, 3GPP/TSGR\#6(99)932.

[6] Q. Zhang, M. C. Chuah, and O. C. Yue, "Enhanced power ramping scheme for UMTS random access channel," Proc. IEEE VTC'99, pp. 2631-2635, Sept. 1999

[7] H. D. Kim, S. H. Wie, and D. H. Cho, "A prioritized random access with discriminative power ramping step size," in Proc. IEEE VTC 2000 Boston, MA, Sept. 2000, pp. 1751-1757.

[8] G. Frank and R. Weber, "Random access scheme for the ETSI/UTRA WCDMA," in Proc. IEEE VTC'99, Houston, TX, May 1999, pp. 1360-1364.

[9] H. Olofsson, M. Gustafsson, and G. Frank, "Performance evaluation of different random access power ramping proposals for the WCDMA system," in Proc. IEEE PIMRC'99, Osaka, Japan, Sept. 1999.

[10] J. Moberg, M. Löfgren, and R. S. Karlsson, "Throughput of the WCDMA random access channel," in Proc. 1st Mobile Communication Summit, Galway, Ireland, Oct. 2000.

[11] "Proposal for RACH Preambles," Motorola and TI, 3GPP/ TSGR\#6(99)893.

[12] M. Schulist and G. Frank, "Link level performance results for a WCDMA random access scheme with preamble power ramping and fast acquisition indication," in Proc. IEEE VTC'99, Amsterdam, The Netherlands, Sept. 1999, pp. 2581-2585.

[13] Y. V. Zakharov, J. F. Adlard, and T. C. Tozer, "Detection of random access burst in W-CDMA system," in Proc. IEEE PIMRC 2000, London, U.K., Sept. 2000, pp. 82-86.

[14] M. Ardebilipour and R. Tafazolli, "A novel implication of reverse link acquisition," in Proc. 3G Mobile Communication Technologies, Elba, Italy, 2000.

[15] H. R. Park and B. J. Kang, "On the performance of a maximum-likelihood code acquisition technique for preamble search in a CDMA reverse link," IEEE Trans. Veh. Technol., vol. 47, pp. 65-74, Feb. 1998.

[16] Y. H. You, Y. C. Lee, T. H. Moon, and C. E. Kang, "Parallel code acquisition technique for preamble search in the CDMA reverse link," Electron. Lett., vol. 32, pp. 1169-1170, June 1996.

[17] B. J. Kang, H. R. Park, M. S. Lim, C. E. Kang, and J. Y. Son, "Access channel slot acquisition in cellular DS/CDMA reverse link," in Proc. IEEE VTC'96, Atlanta, GA, Apr. 28-May 1 1996, pp. 1453-1457.

[18] E. A. Sourour and S. C. Gupta, "Direct-sequence spread spectrum parallel acquisition in a fading mobile channel," IEEE Trans. Commun., vol. 38, pp. 992-998, July 1990.

[19] W. C. Jakes, Microwave Mobile Communications. New York: Wiley, 1974.

[20] A. Polydoros and C. L. Weber, "A unified approach to serial search spread-spectrum code acquisition-Part II: A matched filter receiver," IEEE Trans. Commun., vol. COM-32, pp. 550-560, May 1984.

[21] C.-C. Tseng, "Design and Analysis of Pseudo-Noise Code Acquisition Techniques in Spread Spectrum Systems," Ph.D. disseratation, Nat. Chung Cheng Univ., Taiwan, 2001.
[22] A. J. Viterbi, CDMA: Principles of Spread Spectrum Communication Reading, MA: Addison-Wesley, 1995.

[23] J. G. Proakis, Digital Communication. New York: McGraw-Hill, 1995.

[24] M. Schwartz, W. R. Bennett, and S. Stein, Communication Systems and Techniques. New York: McGraw-Hill, 1966. Appendix A.

[25] C. W. Helstrom, Elements of Signal Detection and Estimation. Englewood Cliffs, NJ: Prentice-Hall, 1995.

[26] M. J. Maron and R. J. Lopez, Numerical Analysis. Belmont, CA: Wadsworth, 1991

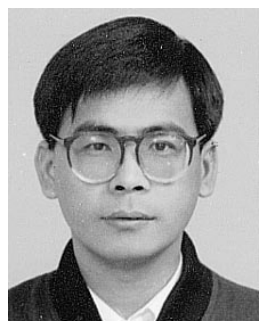

Wern-Ho Sheen (M'91) received the B.S. degree from the National Taiwan University of Science and Technology, Taipei, Taiwan, R.O.C., in 1982, the M.S. degree from the National Chiao Tung University, Hsinchu, Taiwan, R.O.C., in 1984, and the Ph.D. degree from Georgia Institute of Technology, Atlanta, in 1991.

From 1993 to 2001, he was with the National Chung Cheng University, Taiwan, R.O.C, where he held positions as Professor in the Department of Electrical Engineering and Managing Director of the Center for Telecommunication Research. Since 2001, he has been a Professor in the Department of Communication Engineering, National Chiao Tung University, Hsinchu, Taiwan, R.O.C. His research interests include general areas of communication theory, cellular mobile, and personal radio systems, adaptive signal processing for wireless communications, spread spectrum communications, and VLSI design for wireless communications systems.

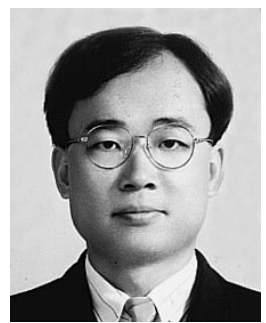

Chun-Chieh Tseng received the B.S. degree in electronics from the National Taiwan Institute of Technology, Taipei, Taiwan, R.O.C., in 1992, and the M.S.E.E. and Ph.D. degrees from the National Chung Cheng University, Chia-Yi, Taiwan, R.O.C., in 1996 and 2001, respectively.

In 2001, he joined the Syncomm Technology Corporation, Taoyuan, Taiwan, R.O.C., where he is currently involved in the project of wireless LAN system and in charge of system level and inner receiver design. His research interests include personal and mobile radio systems, spread spectrum communications, and OFDM-based systems.

Jan-Shin Ho (S'01) received the B.S. degree in communication engineering from the National Chiao Tung University, Hsinchu, Taiwan, R.O.C., in 1993 and the M.S.E.E. degree from the National Chung Cheng University, Chia-Yi, Taiwan, R.O.C., in 1997. Currently, he is working toward the Ph.D. degree in electrical engineering at the National Chung Cheng University.

His research interests include the inner receiver design of the spread spectrum communication systems, personal mobile radio systems, and synchronization in digital receiver. 